\title{
Spherical aberration and its correction using Lie algebraic methods
}

\author{
GOVINDAN RANGARAJAN* and MINITA SACHIDANAND \\ Department of Mathematics, Indian Institute of Science, Bangalore 560012, India \\ *Also at Centre for Theoretical Studies, Indian Institute of Science, Bangalore 560012, India \\ Email: rangaraj@math.iisc.ernet.in
}

MS received 7 August 1997; revised 18 September 1997

\begin{abstract}
We discuss three methods to correct spherical aberration for a point to point imaging system. First, results obtained using Fermat's principle and the ray tracing method are described briefly. Next, we obtain solutions using Lie algebraic techniques. Even though one cannot always obtain analytical results using this method, it is often more powerful than the first method. The result obtained with this approach is compared and found to agree with the exact result of the first method.
\end{abstract}

Keywords. Spherical aberration; Lie algebraic methods.

PACS Nos 42.15; 02.20

\section{Introduction}

In this paper we discuss three different computational methods for correction of spherical aberration in an optical imaging system. The imaging system considered is a lens that focusses rays originating from a single initial point onto a final point. The final point is at the same distance from the centre of the lens as the initial point.

If the above system is assumed to be ideal, as in figure 1 , it would take all light rays originating from point $a$ and focus them onto the focal point $b$ irrespective of the angles these rays make with the optic axis. If the system is nonideal (as it would be in general), the above situation is obtained only in the Gaussian or paraxial approximation. In the non-Gaussian regime, rays are displaced away from the focal point. In particular, if spherical aberration [1] is present, a given ray is displaced in the transverse direction away from the paraxial focus. The magnitude of this displacement is dependent on the angle the ray makes with the optic axis.

Our system is taken to be symmetric about a plane through the origin perpendicular to the optic axis. This enables us to split the system in two right down the middle, and to consider the equivalent but simpler system shown in figure 2 . Our goal is to find the equation for the curved surface of the lens (in figure 2) such that all rays come to a sharp focus at the point $b$ irrespective of the angles they make with the optic axis. Then, this imaging system would be free of on-axis spherical aberration. 


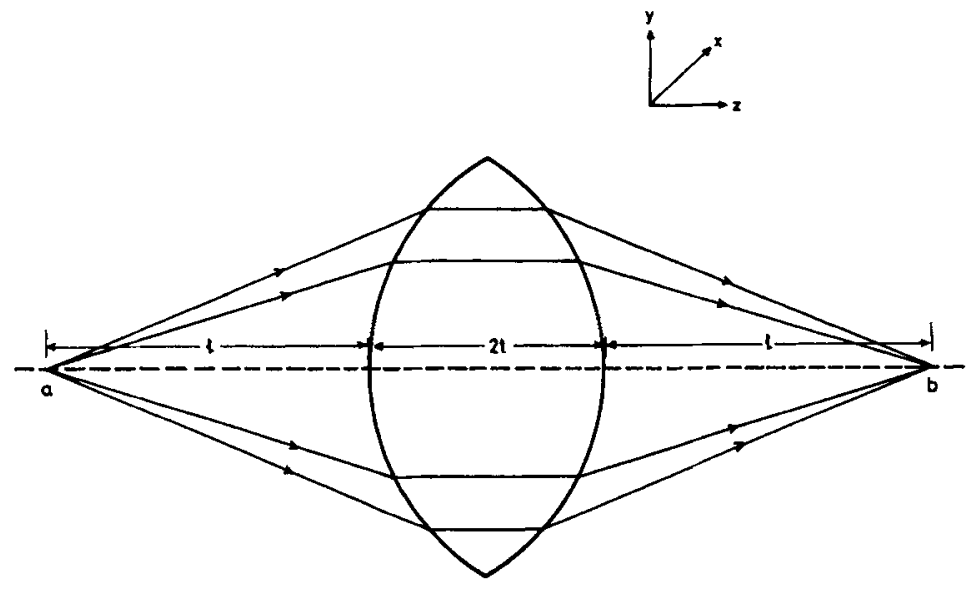

Figure 1. An ideal point-to-point imaging system. It takes all light rays originating at a point $a$ and focusses them onto the focal point $b$. Points $a$ and $b$ are equidistant (at a distance $t+l$ ) from the centre of the lens. The thickness of the lens along the optic axis is $2 t$. Our choice of coordinate system is also indicated in the figure.

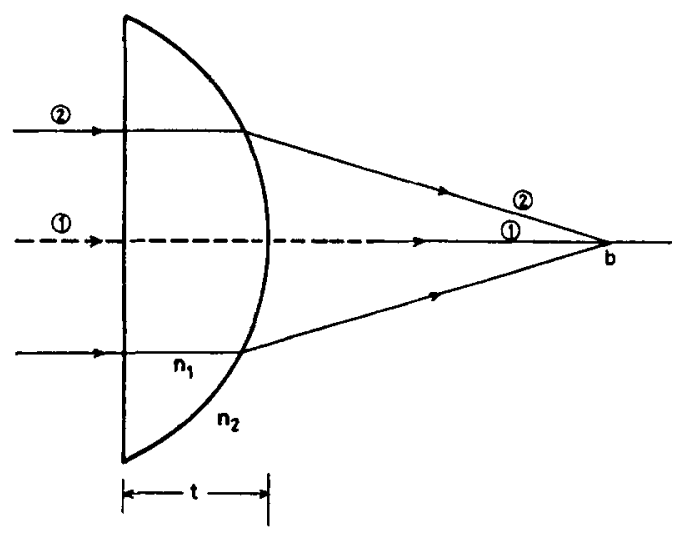

Figure 2. A simpler imaging system equivalent to the original one given in figure 1 . Refractive index $n_{2}=1$ in our case.

In the next section, we directly use Fermat's principle to derive the known analytic expression [1] for the surface of the lens. However, such analytic expressions are possible only for special systems. In the subsequent sections, we compute the surface using more powerful methods that can be used to obtain solutions systematically for general optical systems. In the present case, the exact solution allows us to benchmark solutions obtained using the latter methods. We also briefly describe the result using the method of ray tracing.

In $\S 3$, we introduce the Lie algebraic treatment of optical systems. This method is applied to the problem at hand in $\S 4$, and the results are shown to agree with the exact solution. We give our conclusions in the final section. 


\section{Spherical aberration and its correction}

\section{Exact solution using Fermat's principle}

In this section, we derive the equation for the surface of the lens using Fermat's principle. For our optical system (see figure 2), this principle states that rays 1 and 2 should have the same optical path length. Thus (in the $y-z$ plane)

$$
A=n(t+z)+\sqrt{(l-z)^{2}+y^{2}}=n t+l
$$

where $n$ is the index of refraction of the lens, $t$ is its thickness along the optic axis and $l$ is its focal length.

Solving the above equation, we obtain $z$ as a function of $y$ (we pick the solution where $z$ decreases as $y$ increases, this being the physically appropriate solution for our problem):

$$
z=\frac{l}{n+1}\left[1-\sqrt{1+\frac{(n+1) y^{2}}{(n-1) l^{2}}}\right] .
$$

In fact, this is an equation for a hyperbola. Expanding the square root, we get

$$
z=-\frac{y^{2}}{2 l(n-1)}+\frac{(n+1) y^{4}}{8 l^{3}(n-1)^{2}}-\frac{(n+1)^{2} y^{6}}{16 l^{5}(n-1)^{3}}+\frac{5(n+1)^{3} y^{8}}{128 l^{7}(n-1)^{4}}+\cdots
$$

Thus we have succeeded in obtaining the exact shape of a lens such that our point-topoint imaging systems are free of on-axis spherical aberration. This solution will enable us to verify the correctness of results obtained using the Lie algebraic methods.

One can rederive the equation for the surface of the lens using the method of ray tracing. Employing Snell's law, the following differential equation satisfied by the surface of the lens can be obtained as

$$
\frac{\mathrm{d} z}{\mathrm{~d} y}=\frac{y}{(l-z)-n\left[(l-z)^{2}+y^{2}\right]^{1 / 2}} .
$$

The above differential equation can be solved numerically to obtain the equation for the surface of the lens. It can also be easily verified that the exact solution derived earlier [cf. eq. (2.2)] satisfies this differential equation.

\section{Lie algebraic techniques in optics}

In this section, we consider the application of Lie algebraic techniques to a general optical system $[2,3]$ with the optic axis oriented along the $z$-direction. We assume the optical system to be axially symmetric about the $z$-axis and also symmetric with respect to reflections through some plane containing the $z$-axis.

The optical path length $A$ of a general ray going from the plane $z^{i}$ to the plane $z^{f}$ is defined as follows:

$$
A=\int_{a}^{b} n(r) \mathrm{d} s
$$

where $n(r)$ is the index of refraction at a point $r$ and $s$ is the distance parameter along a curve joining points $a$ and $b$. Taking $z$ to be the independent variable, the distance 


\section{Govindan Rangarajan and Minita Sachidanand}

function ds (in $A$ ) now becomes

$$
\mathrm{d} s=\left(\mathrm{d} x^{2}+\mathrm{d} y^{2}+\mathrm{d} z^{2}\right)^{1 / 2}=\left(1+x^{2}+y^{2}\right)^{1 / 2} \mathrm{~d} z
$$

where the prime denotes differentiation with respect to $z$. Thus

$$
A=\int_{z^{i}}^{z^{f}} n(x, y, z)\left(1+x^{2}+y^{2}\right)^{1 / 2} \mathrm{~d} z .
$$

Comparing this with the action functional in mechanics [4], the integrand in the expression for $A$ can be identified as the Lagrangian $L$ for our system

$$
L=n(x, y, z)\left(1+x^{2}+y^{2}\right)^{1 / 2}
$$

We can also define the conjugate momenta:

$$
\begin{aligned}
& p_{x}=\frac{\partial L}{\partial x^{\prime}}=n(x, y, z) \frac{x^{\prime}}{\left[1+x^{2}+y^{2}\right]^{1 / 2}}, \\
& p_{y}=\frac{\partial L}{\partial y^{\prime}}=n(x, y, z) \frac{y^{\prime}}{\left[1+x^{2}+y^{2}\right]^{1 / 2}} .
\end{aligned}
$$

Apart from the factor of $n$, these are just the direction cosines of the ray. Using these, we obtain the Hamiltonian in the usual way:

$$
H=-\left[n^{2}(x, y, z)-p_{x}^{2}-p_{y}^{2}\right]^{1 / 2}
$$

In Lie perturbation theory, instead of solving the Hamilton's equations of motion directly, one solves the problem in the following map form

$$
w^{f}=\mathcal{M} w^{i},
$$

where $\mathcal{M}$ is a nonlinear symplectic map mapping the initial values of the phase-space 4vector $w=\left(x, y, p_{x}, p_{y}\right)$ to its final values. For further details see ref. [5]. There is a standard procedure to obtain $\mathcal{M}$ from the Hamiltonian which is described elsewhere [6]. It can be further shown that $\mathcal{M}$ can be factorized as follows:

$$
\mathcal{M}=e^{: f_{2}:} e^{: f_{3}:} e^{: f_{4}: \ldots e} e^{: f_{n}: \ldots},
$$

where $f_{m}$ 's are homogeneous polynomials of degree $m$ in the phase space variables. The quantity $: f_{m}$ : is the Lie operator associated with the function $f_{m}$ and is defined by its action on a general phase-space function $g$ as follows:

$$
: f_{m}: g=\left[f_{m}, g\right]
$$

where [,] denotes the usual Poisson bracket. Finally, we define the Lie transformation associated with the Lie operator $: f_{m}:$ as

$$
\exp \left(: f_{m}:\right)=\sum_{n=0}^{\infty} \frac{: f_{m}:^{n}}{n !} \text {. }
$$

It can be shown $[2,3]$ that the various $f_{m}$ 's occuring in the factored form of $\mathcal{M}$ have a definite physical interpretation for an imaging system. The $f_{2}$ 's represent paraxial 


\section{Spherical aberration and its correction}

optics, the $f_{3}$ 's are related to second order aberrations, the $f_{4}$ 's to third order aberrations and so on.

We end this section by writing down the symplectic map for two systems which will be used later. First, consider the simple case of transit through a slab of thickness $l$ with refractive index $n$. It can be easily shown that the symplectic map corresponding to this system is given by

$$
\mathcal{M}=\exp \left[l:\left(n^{2}-p^{2}\right)^{1 / 2}\right]
$$

where $p=\left(p_{x}, p_{y}\right)$. In the factorized form, this is given as

$$
\begin{aligned}
\mathcal{M}= & \exp \left(-\frac{l}{2 n}: p^{2}:\right) \exp \left(-\frac{l}{8 n^{3}}:\left(p^{2}\right)^{2}:\right) \\
& \times \exp \left(-\frac{l}{16 n^{5}}:\left(p^{2}\right)^{3}:\right) \exp \left(-\frac{5 l}{128 n^{7}}:\left(p^{2}\right)^{4}:\right) \cdots,
\end{aligned}
$$

where $p^{2}=p_{x}^{2}+p_{y}^{2}$. In order to verify this equation, note that

$$
\begin{aligned}
& p^{f}=\mathcal{M} p^{i}=p^{i}, \\
& q^{f}=\mathcal{M} q^{i}=q^{i}+l p^{i} /\left[n^{2}-\left(p^{i}\right)^{2}\right]^{1 / 2},
\end{aligned}
$$

where $q=(x, y)$. Using (3.5) in the above equations we get

$$
p^{f}=p^{i}, \quad q^{f}=q^{i}+l\left(q^{\prime}\right)^{i} .
$$

Remembering that $q^{\prime}=\mathrm{d} q / \mathrm{d} z$, we see that this is just what would be expected for transit through a distance $l$ along the $z$-axis.

Note that only even powers of $p$ occur in the expression for $\mathcal{M}$ in (3.12). This is in fact true for all optical systems having the assumed axial and reflection symmetries. These symmetries imply that only the phase space variables $p^{2}, p \cdot q$ and $q^{2}$ (and their various combinations and powers) can occur in $f_{m}$ 's. This automatically implies that only even degree homogeneous polynomials i.e. $f_{2}, f_{4}$, etc. are allowed.

Next, we consider propagation of a light ray through the system given in figure 3 . We again assume axial and reflection symmetries. The surface of the interface is parametrized by the equation

$$
z^{s}=\beta_{2}\left(x^{2}+y^{2}\right)+\beta_{4}\left(x^{2}+y^{2}\right)^{2}+\beta_{6}\left(x^{2}+y^{2}\right)^{3}+\beta_{8}\left(x^{2}+y^{2}\right)^{4}+\cdots
$$

After some computation, one finds the $\mathcal{M}$ (truncated to order 8 ) for such a surface to be

$$
\mathcal{M}=e^{: f_{2}:} e^{: f_{4}:} e^{: f_{6}:} e^{: f_{8}:}
$$

where

$$
f_{2}=\beta_{2}\left(n_{1}-n_{2}\right) q^{2}
$$

and

$$
\begin{aligned}
f_{4}= & -\beta_{2}^{3} \frac{\left(n_{1}-n_{2}\right)}{n_{1}}\left[n_{1}\left(2-\frac{\beta_{4}}{\beta_{2}^{3}}\right)-2 n_{2}\right]\left(q^{2}\right)^{2} \\
& +2 \beta_{2}^{2} \frac{\left(n_{1}-n_{2}\right)}{n_{1}} q^{2}(p \cdot q)+\frac{\beta_{2}\left(n_{1}-n_{2}\right)}{2 n_{1} n_{2}} q^{2} p^{2} .
\end{aligned}
$$




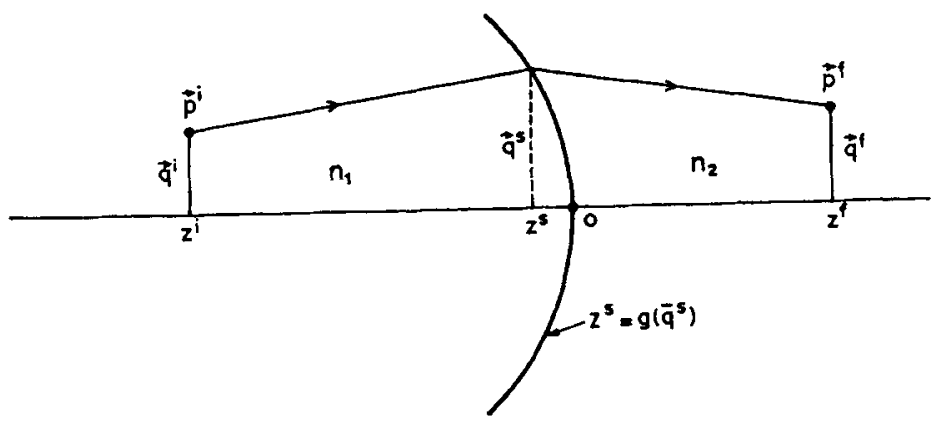

Figure 3. An (axially symmetric) optical system consisting of an interface separating media of refractive indices $n_{1}$ and $n_{2}$. A ray originating at $z^{i}$ with initial conditions $q^{i}$ and $p^{i}$ passes through the interface and terminates at $z^{f}$ with final conditions $q^{f}$ and $p^{f}$. The interface is parametrized by the equation $z^{s}=g\left(q^{s}\right)$.

The expressions for $f_{6}$ and $f_{8}$ are too long to be documented here. Since we have truncated $\mathcal{M}$ to order 8 , the error made in using this truncated expression is also of order 8 .

\section{Correction of spherical aberration using Lie algebraic method}

Before applying the Lie algebraic method to our problem, we briefly describe our plan of action. We will first construct the symplectic map $\mathcal{M}$ (up to order 8) corresponding to our optical system (see figure 2). Since we include all terms up to $f_{8}$ in $\mathcal{M}$, we would have taken into account all aberrations up to the seventh order. $\mathcal{M}$ will depend on the surface of the lens and hence on $\beta_{2}$ through $\beta_{8}$ [cf. eq. (3.16)]. Next, we will require that our map satisfy a set of conditions which ensure that $\mathcal{M}$ (and hence our system) is free of spherical aberration up to seventh order. Since $\mathcal{M}$ is a function of $\beta_{i}$ 's, this would be equivalent to the statement that these $\beta_{i}$ 's should satisfy certain equations. This will fix our $\beta_{i}$ 's and hence the equation for the surface of the lens to eight order. Finally, we compare this equation with the exact result obtained in $\S 2$ and show that they agree (up to order 8).

Coming back to our problem, we see that our system (figure 2) has three components:

(1) A transit through distance $t$ in a medium of refractive index $n$ (the lens).

(2) The surface of the lens separating media of refractive indices $n$ and 1 (air) respectively.

(3) A transit through distance $l$ (the focal length) in air $(n=1)$.

The symplectic maps $\mathcal{M}_{1}$ and $\mathcal{M}_{3}$ for (1) and (3) are given by expressions of the general form of equation (3.12). The map $\mathcal{M}_{2}$ for (2) is given by equation (3.17). One of the advantages of the Lie algebraic approach now becomes apparent - the combined map $\mathcal{M}$ for the entire system is just the product of the maps for the individual components:

$$
\begin{aligned}
& \mathcal{M}=\mathcal{M}_{1} \mathcal{M}_{2} \mathcal{M}_{3}=\exp \left(-\frac{t}{2 n}: p^{2}:\right) \exp \left(-\frac{t}{8 n^{3}}:\left(p^{2}\right)^{2}\right): \cdots \\
& \exp \left(\beta_{2}(n-1): q^{2}:\right) \exp \left(: f_{4}:\right) \exp \left(: f_{6}:\right) \exp \left(: f_{8}:\right) \cdots
\end{aligned}
$$




$$
\exp \left(-\frac{l}{2}: p^{2}:\right) \exp \left(-\frac{l}{8}:\left(p^{2}\right)^{2}:\right) \exp \left(-\frac{l}{16}:\left(p^{2}\right)^{3}:\right) \exp \left(-\frac{5 l}{128}:\left(p^{2}\right)^{4}:\right) \cdots
$$

Using Lie algebraic manipulation techniques [5], we recast the symplectic map $\mathcal{M}$ into the standard form:

$$
\mathcal{M}=\exp \left(: g_{2}:\right) \exp \left(: g_{4}:\right) \exp \left(: g_{6}:\right) \exp \left(: g_{8}:\right)
$$

where $g_{2}, g_{4}$ etc are of the form

$$
\begin{aligned}
& g_{2}=A_{2} p^{2}+B_{2} p \cdot q+C_{2} q^{2} \\
& g_{4}=A_{4}\left(p^{2}\right)^{2}+B_{4}(p \cdot q) p^{2}+\cdots \\
& g_{6}=A_{6}\left(p^{2}\right)^{3}+B_{6}(p \cdot q)\left(p^{2}\right)^{2}+\cdots \\
& g_{8}=A_{8}\left(p^{2}\right)^{4}+B_{8}(p \cdot q)\left(p^{2}\right)^{3}+\cdots
\end{aligned}
$$

Again, the expressions for $A_{2}, B_{2}$ etc have been omitted for the sake of brevity. These coefficients depend on $l$ and $n$. In addition, the coefficients in $g_{2}$ (i.e. $A_{2}, B_{2}$ and $C_{2}$ ) depend on $\beta_{2}$, the coefficients in $g_{4}$ on $\beta_{2}$ and $\beta_{4}$ and so on.

Now we are ready to determine $\beta_{2}, \beta_{4}, \beta_{6}$ and $\beta_{8}$ appearing in the equation for the surface of the lens [cf. eq. (3.16)]. We know that $g_{2}$ in $\mathcal{M}$ represents paraxial optics. In the paraxial approximation, it is clear from figure 2 that we want rays that start out parallel (i.e. $w^{i}=(q, 0)$ ) to come to a focus at a distance $l$ along the z-axis (i.e. $\left.w^{f}=(0, p)\right)$. Thus,

$$
\left(\begin{array}{l}
0 \\
p
\end{array}\right)=e^{: 8_{2}:}\left(\begin{array}{l}
q \\
0
\end{array}\right)
$$

Since $l$ and $n$ in $g_{2}$ are already given, this equation fixes the only other free parameter $\beta_{2}$ to be

$$
\beta_{2}=\frac{1}{2 l(1-n)}
$$

We fix $\beta_{4}, \beta_{6}$, and $\beta_{8}$ by imposing conditions which ensure that $\mathcal{M}$ is free of spherical aberration (up to order 7). As defined earlier, spherical aberration is the phenomenon where a given ray is displaced (in the transverse direction) from the paraxial focus on the $z$-axis by an amount dependent only on its arrival angle (i.e. $p$ in our case).

It can be shown that only the $\left(p^{2}\right)^{2},\left(p^{2}\right)^{3}$, and $\left(p^{2}\right)^{4}$ terms (in $g_{4}, g_{6}$, and $g_{8}$ respectively) give rise to spherical aberration of orders 3,5 , and 7 respectively. For example, consider the effect of $\left(p^{2}\right)^{2}$ term in $g_{4}$ :

$$
q^{f}=\exp \left(: A_{4}\left(p^{2}\right)^{2}:\right) q=q+A_{4}\left[\left(p^{2}\right)^{2}, q\right]=q-4 A_{4}\left(p^{2}\right) p
$$

Thus,

$$
\delta q=q^{f}-q=-4 A_{4}\left(p^{2}\right) p
$$

Hence the displacement of the actual arrival point $q^{f}$ from the Gaussian arrival point $q$ depends only on its Gaussian arrival direction $p$. This is indeed spherical aberration. 


\section{Govindan Rangarajan and Minita Sachidanand}

Evidently to eliminate spherical aberration we need to make $\delta q$ zero. In our case, we can eliminate it up to order 7 by setting

$$
A_{4}=A_{6}=A_{8}=0 \text {. }
$$

We can now solve these equations for $\beta_{4}, \beta_{6}$, and $\beta_{8}$ in terms of $n$ and $l$. The resulting expressions (after some manipulation) are

$$
\begin{aligned}
& \beta_{4}=\frac{(n+1)}{8 l^{\beta}(n-1)^{2}}, \\
& \beta_{6}=-\frac{(n+1)^{2}}{16 l^{5}(n-1)^{3}}, \\
& \beta_{8}=\frac{5(n+1)^{3}}{128 l^{7}(n-1)^{4}} .
\end{aligned}
$$

Thus we have succeeded in determining the shape of the lens surface such that our imaging system is free of on-axis spherical aberration (to seventh order). In the $y-z$ plane, this is given by [after substituting the above expressions into eq. (3.16)]

$$
z^{s}=-\frac{y^{2}}{2 l(n-1)}+\frac{(n+1) y^{4}}{8 l^{3}(n-1)^{2}}-\frac{(n+1)^{2} y^{6}}{16 l^{5}(n-1)^{3}}+\frac{5(n+1)^{3} y^{8}}{128 l(n-1)^{4}}
$$

We see that this agrees exactly with the equation obtained in $\S 2$ [cf. eq. (2.3)] up to the calculated order.

\section{Conclusion}

In this paper, we discussed the problem of eliminating on-axis spherical aberration from a point-to-point imaging system. We solved the problem using three methods-direct application of Fermat's principle, ray tracing method, and Lie algebraic method. The results were shown to agree. A direct application of Fermat's principle, even though it gives the exact result in this particular case, is not possible for most optical systems. In contrast, Lie algebraic methods can be applied to a wide variety of optical systems. In particular, they can be applied to systems with no particular symmetry and systems with alignment and positioning errors. They can also be used to analyse graded index systems. All this is possible because the mapping from initial to final conditions in any optical system can be shown [3] to be a symplectic mapping. And symplectic maps can be analysed very efficiently using Lie algebraic methods. In fact, Lie algebraic methods have applications in other fields also [7-10].

\section{Acknowledgements}

The authors would like to thank Prof. Alex Dragt for helpful discussions. This work was supported in part by the Department of Science and Technology, Research Grant No. $\mathrm{SP} / \mathrm{S} 2 / \mathrm{K}-28 / 96$. 


\section{References}

[1] R K Luneberg, Mathematical theory of optics (University of California Press, Berkeley, 1964)

[2] A J Dragt, J. Opt. Soc. Am. 72, 372 (1982)

[3] A J Dragt, E Forest and K B Wolf, in Lie methods in optics edited by J S Mondragon and K B Wolf (Springer Verlag, Berlin, 1986)

[4] H Goldstein, Classical mechanics, second edition (Addison-Wesley, Reading, 1980)

[5] A J Dragt, F Neri, G Rangarajan, D R Douglas, L M Healy and R D Ryne, Ann. Rev. Nucl. Part. Sci. 38, 455 (1988) and references therein

[6] A J Dragt and E Forest, J. Math. Phys. 24, 2734 (1983)

[7] G Rangarajan, J. Math. Phys. 37, 4514 (1996); Pramana - J. Phys. 48, 129 (1997)

[8] G Rangarajan, J. Math. Phys. 38, 2710 (1997)

[9] F Neri and G Rangarajan, Phys. Rev. Lett. 64, 1073 (1990)

[10] A J Dragt, F Neri and G Rangarajan, Phys, Rev. A45, 2572 (1992) 